\title{
Inflammation, Osteoporosis and Atherosclerosis: The Tsimtsoum Concept
}

\author{
Kumar Kartikey, Garima Singh, Ram B. Singh*, Agnieszka Wilczynska and Fabien De Meester
}

Tsim Tsoum Institute, Ul. Golebia 2, 31-007, Krakow, Poland

\begin{abstract}
There is evidence that the high intake of $\omega 6$ and low intake of $\omega 3$ fatty acids from the diet contribute to the development of CVD as well as of osteoporotic hip fractures. Increased intake of fruits, vegetables, legumes and nuts that are rich in $\omega 3$ fatty acids (alpha-linolenic acid) has recently been found to be protective against CVD and osteoporosis, which raises the possibility that these foods and their nutrients may protect against CVD and hip fracture, and may provide better quality of life. Increased risks in co-twins without an index diagnosis suggest that genetic factors and genes/diet interactions may explain the association of CVD with osteoporotic fractures. There is evidence that Western diet rich in refined carbohydrates (coke drinks, biscuits, cakes, ice creams), trans fat, high $\omega 6$ / low $\omega 3$ fats, deficiencies in soluble fibers and antioxidants can enhance the expression of about 125 genes, the majority of which are pro-inflammatory; there is increase in AP-1, Egr-1 and NFkB, that are transcription factors for inflammation. It is possible that these pro-inflammatory factors increase risk of osteoporosis as well as of atherothrombosis, resulting into hip fractures and CVD, respectively. Calcification of vascular walls shares similarities with bone formation and resorption processes; in particular, bisphosphonates are known to not only decrease the progression of osteoporosis, but also to prevent the development of atherosclerosis, thereby reducing total mortality rates. On the other hand, cholesterol-lowering statins are anti-inflammatory and reduce risk of CVD and osteoporotic fractures.
\end{abstract}

Keywords: Hip fractures, coronary heart disease, noncommunicable diseases, diet.

\section{INTRODUCTION}

There is convincing evidence for a reduction in risk of osteoporosis and atherosclerosis with increased intake of vitamin D and calcium and physical activity and for an increased risk with high intake of western diet and sedentary behaviour [1-9]. No study has examined the role of nutrients among post operative outcome in patients with osteoporotic hip fracture. Dietary calcium, vitamin D and magnesium deficiency have been studied in the pathogenesis of osteoporosis (known to predispose fractures), as well as in atherosclerosis [4]. However, the role of $\omega-3$ fatty acid metabolism in the pathogenesis of osteoporosis has not been studied thoroughly, although several studies are available to indicate that w-3 fatty acids are cardioprotective and antithrombotic [7-10]. Osteoporosis is a disease affecting many millions of people around the world. It is characterized by low bone mass and micro-architectural deterioration of bone tissue, leading to bone fragility and a consequent increase in risk of fracture [1-3]. The incidents of vertebral and hip fractures increases exponentially with advancing age (while that of risk of fractures levels off after the age of 60 years [3]. Osteoporosis fractures are a major cause of morbidity and disability in older people and, in the case of hip fractures can lead to pre-mature death [1-4]. Such fractures impose a considerable economic burden on health services in both developed and developing countries [4]. Worldwide variation in the incident and prevalence of osteoporosis is difficult

*Address correspondence to this author at the Tsim Tsoum Institute, Ul.Golebia 2, 31-007, Krakow, Poland; Tel/Fax: 0091591 2417437;

E-mail: rbs@tsimtsoum.net to determine because of problems with definition and diagnosis. The most useful way of comparing osteoporosis prevalence between populations is to use fracture rates in older people. However, because osteoporosis is usually not life threatening, quantitative data from developing countries are scarce, despite this, the current consensus is that approximately 1.66 million hip fractures occur each year worldwide, that the incidence is set to increase four-fold by 2050 , because of the increasing numbers of older people, and that the age -adjusted incidence rates are many times higher in affluent developed countries than in sub Saharan Africa and Asia [1-4]. Recent studies [5, 6] indicate that hip fractures are 2-5 fold more common among patients with cardiovascular disease (CVD) which poses the possibility that both problems may have a common unifying hypothesis for their pathogenesis, to explain the cause of this association. The role of $\mathrm{w}-3$ fatty acids and the body-mind interaction in relation to atherosclerosis and osteoporosis need further consideration.

\section{OSTEOPOROSIS, ATHEROSCLEROSIS AND DIET AND LIFESTYLE FACTORS}

The last few decades of the last century greatly increased our knowledge about the global dimensions of the noncommunicable diseases (NCD) including osteoporosis and CVDs [1-8]. Recent studies indicate that there is coexistence of nutritional deficiencies and appreciable overnutrition in the form of central obesity and overweight in developing countries and in developed countries one quarter of the population is obese, which may be risk factors for CVD and osteoporosis [1-6]. Hip fractures rates are highest in Caucasian 
women living in temperate climate, slightly lower in women from Mediterranian and Asian countries, and are lowest in women in Africa [1-4]. The lower incidence of hip fractures in these countries indicate that differences in diet and lifestyle appear to be important in the pathogenesis and prevention of osteoporosis and hip fractures, similar to atherosclerosis [9-13]. Experimental and clinical studies [13-16] indicate that the high intake of w-6 and low w-3 fatty acids in the diet contribute to the development of CVD as well as osteoporotic hip fractures. Recently, increased intake of fruits, vegetables, legumes, fish and nuts that are rich in w-3 fatty acids (alpha linolenic acid) have been found to be protective against risk of CVD and osteoporosis, which poses the possibility that these foods and nutrients may protect against fracture and CVD and provide better quality of life [13-20]. High protein diet and physical activity also protect against osteoporosis induced fractures as well as against CVD. These protective factors are also beneficial against depression and memory disfunction indicating that body mind interaction appears to be important in the prevention of these diseases.

It has been proposed that overweight comes first in conjunction with inflammation, hyperinsulinemia, increased angiotensin activity, vascular variability disorders and central obesity followed by glucose intolerance, type 2 diabetes, hypertension, low HDL and hypertriglyceridemia (Metabolic syndrome) $[9,10]$.This sequence is followed by CAD, gall stones and cancers and finally dental caries, gastrointestinal diseases, degenerative diseases of the brain and psychological disorders, and bone and joint diseases. We suggest that this sequence also includes osteoporosis, during transition from poverty to affluence. These NCDs may be associated with increased production of thromboxane A2, leucotrienes, interleukins- 1 and 6 , tumor necrosis factor-alpha and Creactive protein. Increased dietary intake of $w-6$ fatty acids and low w-3 fatty acids are known to enhance these risk factors and risk of all these diseases, by having adverse proinflammatry effects, whereas increased w-3 fatty acids are protective [9-20].

There is evidence [21], that depression and dementia may be associated with atherosclerosis as well as osteoporosis because of a unifying hypothesis that inflammation characterized with high concentrations of C-reactive protein, IL-6, IL-1 and TNF-alpha can predispose all the three chronic diseases of affluence.

\section{OSTEOPOROSIS AND CARDIOVASCULAR DISEASE}

In one study [6], clinical and radiological examination of 25 patients (40-70 years) showed that, half of the subjects were men. Prosthetic surgery; Austin Moores Prosthesis and Bipolar Prosthesis were used in all the patients who were hospitalized and followed up daily for various complications during hospitalization and at weekly follow up for 12 weeks. Quality of life was assessed by a 6 min walk test and the distance walked was recorded. We recorded 4 hourly blood pressures, heart rate, respiratory rate and temperature to study their time adjusted variations for early diagnosis of infections, and vascular complications. One fifth of the patients had indicating high risk of CVD. Of total 25 patients, one patient developed acute myocardial infarction and an- other one stroke. Popliteal vein thrombosis and thromboflebitis were observed in approximately one third of the patients. Most vascular injuries have been reported to occur during revision surgery and since there is an increase in the number of revisions being performed, the incidence of vascular injuries may increase. Subjects taking statins may have less osteoporosis and less vascular complications. Approximately $95 \%$ patients had osteoporosis and osteoporosis was the commonest cause of hip joint fracture which was associated with low intake of protein in the diet and sedentary behaviour. Larger studies would be necessary to find out the incidence of vascular complications of hip joint surgery and its association with CVDs.

In a most recent cohort study [5], it has been suggested by Synnerby and colleagues that clinicians should be aware of the considerably increased rate of hip fracture in both sexes, especially after a recent hospitalization for CVD. Osteoporosis and CVD are prevalent in elderly individuals, but until recently have been regarded as independent age-related disorders. Recent research indicate at commonalities between the disorders - for example, bone and vasculature are regulated by several shared factors, in which calcification of the vascular walls in many ways resembles the bone formation and bone resorption process. Similarly, bisphosphonates are known to decrease the progression of osteoporosis, as well as also prevent the development of atherosclerosis and reduce total mortality rate; while cholesterol-lowering statins that reduce risk of CVD are thought to reduce the risk of osteoporotic fractures.

In the same study [5], the researchers examined data from the Swedish Twin Registry which contains 31,936 twin members born between 1914 and 1944, who were followedup from the time of their 50th birthday. By examining the National Patient Registry, the investigators found that individuals with CVD had a significantly increased prevalence of hip fractures than those with no CVD. The crude absolute rate of hip fractures in individuals without a history of CVD was 1.2 per 1000 person-years. This compared with a rate of 12.6 after a diagnosis of heart failure, 12.6 after a diagnosis of stroke, 6.6 after a diagnosis of peripheral atherosclerosis, and 5.2 after a diagnosis of ischemic heart disease. Corresponding hazard ratios (HR) for hip fracture after a diagnosis of CVD were 5.09, 4.40, 3.20, and 2.32 for stroke, heart failure, peripheral atherosclerosis, and ischemic heart disease, respectively, compared with patients with no CVD. The researchers then looked at identical twin pairs where one cotwin had CVD and the other did not. They found that the unaffected co-twins had a significantly increased risk for hip fracture when compared with unaffected twin pairs, at HRs of 3.74 for "pseudo exposure" to heart failure and 2.29 for pseudo exposure to stroke. The authors pointed out that most of the overall increased rate of hip fracture after heart failure (and part of the increased risk after stroke) appears to be explained by genes or by early environmental sharing (ie, not individual lifestyle habits or other individual-specific environmental factors). However, it is known that environmental factors can enhance the expression of genes responsible for CVD, heart failure and osteoporosis due to increased consumption of w-6 fat, trans fat and refined carbohydrates.

According to a further study [8], the associations of food consumption pattern and w-6/w-3 fatty acid ratio of the diet 
were examined with hip joint fractures. Sixty cases having fracture neck of femur and 95 control subjects above 50 years of age were included in this case control study. Dietary intakes were obtained by 3 days assessment of food intakes by questionnaires among patients with fractures $(n=50)$ as well as among 95 control subjects. Physical activity was assessed by questionnaires. Radiological and clinical examinations were conducted for the diagnosis of fractures. Cytokines were measured by chemoluminescence enzyme immunometric assay (immulite automated analyzer) kit (DPC Los Angelis, CA, USA). Regression analysis was done to find out the association of risk factors with hip fractures. Among 60 cases, the fracture was more common in male than female. Fruits, vegetables and legume (165 \pm 12.6 vs. $205 \pm 15$. $8 \mathrm{~g} /$ day, $\mathrm{P}<0.03$ ) as well as milk products (milk, curd, butter etc) consumption $(205+25.8$ vs. $318 \pm 31.5 \mathrm{~g} /$ day, $\mathrm{P}<0.05)$ were significantly lower and $\mathrm{w}-6$ rich oils intake was significantly higher among patients with fractures compared to control subjects, respectively. Omega-3 fatty acids intakes were significantly lower among patients with fractures $(0.45 \pm 0.74 \mathrm{~g} / \mathrm{day}, \mathrm{P}<0.05)$. Osteoporosis $(92.0 \%)$, trivial trauma $(92.0 \%)$, physical inactivity $(80.0 \%)$, diabetes mellitus $(21.6 \%)$ were common among patients with hip fracture. Multivariate logistic regression analysis showed that the intakes of fruit, vegetable and legume (odds ratio 1.12, confidence interval (CI) 1.02-1.21, $\mathrm{P}<0.05$ ), physical activity(OR 1.36, CI 1.22-1.52, P<0.05), w-3 fatty acids (OD1.05, 0.92$1.17, \mathrm{P}<0.01)$ intake were inversely associated with fracture, whereas $\mathrm{w}-6 / \mathrm{w}-3$ ratio (OD 1.33, CI 1.18-1.47,P<0.01) interleukin-6, (OD1.11, CI 1.02-1.19, $\mathrm{P}<0.01$ ), tumor necrosis factor-alpha(OD,1.09, CI 1.01-1.17, $\mathrm{P}<0.01)$ were positively associated with fracture. This study showed that increased consumption of fruit, vegetable and legume, milk products and $w-3$ fatty acid and low $w-6 / w-3$ ratio diet as well as physical activity may be protective against hip joint fractures. In a single blind, randomized trial, the interactions of calcium, dihomogamalinolenic acid (DGLA) + eicosapentaenoic acid (EPA) was examined compared to coconut oil+calcium in women with osteoporosis. All subjects were living in a institution for elderly and fed low calcium, nonvitamin D enriched foods and similar exposure to sunlight. Markers of bone formation or degradation and bone mineral density (BMD) were measured at baseline, 6, 12 and 18 months duration. After 18 months, osteocalcin and deoxypyridinoline levels decreased significantly in both groups, indicating a reduction in bone turnover, whereas bone specific alkaline phosphatase increased indicating beneficial effects of calcium given to all the patients. Lumber and femoral BMD showed that lumber spine density remained the same in the treatment group, but decreased $3.2 \%$ in the placebo group. Femoral bone density increased $1.3 \%$ in the treatment group but decreased $2.1 \%$ in the placebo group. During the second period of 18 months with all women receiving intervention treatment, lumber spine density increased $3.1 \%$ among patients who remained on active intervention and $2.3 \%$ among those women who switched from placebo to active treatment; femoral BMD in the latter group showed an increase of $4.7 \%$.

In another study by Terano, 40 elderly women with age related osteoporosis were divided into four groups. They were administered daily for 16 weeks one of 4 treatment regimens in each group; $4 \mathrm{~g}$ evening primrose oil, $4 \mathrm{~g}$ fish oil,
$4 \mathrm{~g}$ of a fish and evening primrose oil mixture or $4 \mathrm{~g}$ olive oil placebo, while no other medication/ supplements or special foods was taken during the trial. In this study, fish oil increased serum calcium, osteocalcin and collagen and decreased ALP. Evening primrose oil alone had no significant effects, but the positive results from the fish oil group were also seen in the fish oil plus evening primrose oil group. It is possible that evening primrose oil may have synergestic effects with fish oil. Evening primrose oil also contains ALA which is metabolized in our body into EPA and docosahaxaenoic acid (DHA) that are long chain w-3 fatty acids present in fish oil.

There is evidence that depression may be associated with reduced bone mineral density (BMD) [21]. In this study, 24 women with a history of major depression were compared with another 24 age, race and body mass index, postmenopausal status matched women controls. There was a significant lower BMD in patients with depression compared to controls. Deficiency of w-3 fatty acids may be a common link between depression and osteoporosis among these subjects, both prevalent among elderly population. These studies $[8,18,19,21]$ indicate that w-3 fatty acids appear to be important in bone health and in the prevention of osteoporosis and support our findings that increased ratio of w-6/w-3 fatty acids or deficiency of $\mathrm{w}-3$ fatty acids may be a risk factor for osteoporosis and hip fractures.

Further support for the role of polyunsaturated fatty acids in the pathogenesis of osteoporosis and hip fracture may be provided from the experimental studies [22-25]. In a experimental study [22], 15 rats were fed identical diets except that w-6/w-3 ratio differed. Safflower oil and fish oil were mixed to produce $\mathrm{w}-6 / \mathrm{w}-3$ ratios of $23.8,9.8,2.6$ and 1.2 . There was a significant increase in PGE2 and ALP with decrease in $\mathrm{w}-6 / \mathrm{w}-3$ ratio in the rat liver and bone tissue [22]. The high concentration of ALP indicated better reabsorption of bone and moreover $1.2 \mathrm{w}-6 / \mathrm{w}-3$ ratio diets showed higher rate of bone formation. In another study [23], an increased production of PGE2 in tibia of chicks given a semipurified diet containing soya bean oil, high in w-6 fat was associated with a lower rate of bone formation compared with that of chicks fed a low dietary ratio of $w-6 / w-3$ fatty acids. In a further study [24], rats fed with a lower dietary ratio of $w-6 / w-3$ fat showed increased bone marrow cellularity and bone strength. These studies indicate that $w-6 / w-3$ fatty acid ratio of diet appears to be important in bone metabolism, and may be responsible for fracture due to trivial trauma in our patients.

These studies provide further proof that dietary fatty acids modulate the fatty acid composition and PGE2 production in these tissues by altering fatty acid composition of membrane phospholipids resulting into improvement in cell function. The bone is highly active metabolic tissue that continues to change throughout life. Bone remodeling occurs up to $20 \%$ which is the process of bone growth associated with maintaining a fixed adult bone mass. Older bone tissue is destroyed (reabsorbed) and replaced by new bone tissue in a cyclical process. However, in osteoporosis, the basic cause is that reabsorption becomes greater ahead of bone formation, resulting in a net bone loss. Diets higher inw-6/w-3 fatty acids are associated with greater PGE2 which have adverse effects [22-24]. Whereas low $\mathrm{w}-6 / \mathrm{w}-3$ ratio diet may provide low PGE2 which stimulates bone formation by increased 
production of insulin like growth factor, which is a powerful growth stimulator for bone and muscle. The growth may be further enhanced by simultaneous treatment with coenzyme Q10 which is potent antioxidant and bioenergetic agent present in the mitochondria of muscles. PGE2 also mediate the effects of vitamin D, TNF-alpha and growth factors which are known to enhance bone reabsorption which results into osteoporosis [22-25].There was a positive association of proinflammatory cytokines; IL-6 and TNF-alpha in patients with hip fracture, in presence of w-3 fatty acids in the diet. Therefore it is possible that w-3 fatty acids modulates PGE2 as well as proinflammatory cytokines; TNF-alpha and IL-6 which are known to enhance PGE2 [25], resulting in to marked increase in bone reabsorption.

\section{THE TSIM TSOUM CONCEPT}

It seems that inflammation is important in the pathogenesis of osteoporosis, atherosclerosis as well as depression and dementia and these problems are related to diet and lifestyle factors which are known to predispose body-mind interactions. Our genes appear to be similar to the genes of our ancestors during the Paleolithic period 40,000 years ago, the time when our genetic profile was established. Man appears to live in an nutritional environment which completely differs from that for which our genetic constitution was selected. However, it was only during the last 100-160 years that dietary intakes have changed significantly, causing increased intake of saturated fatty acids (SFA) and linoleic acid, and decrease in w-3 fatty acids, from grain fed cattle, tamed at farm houses, rather than meat from running animals. There is marked reduction in consumption of w-3 fatty acids, vitamins and minerals and proteins and significant increase in the intakes of carbohydrates, (mainly refined,), fat ( saturated, trans fat, linoleic acid) and salt compared to Paleolithic period.

The Columbus concept of diet means that humans evolved on a diet that was low in saturated fat and the amount of w-3 and w-6 fatty acids was quite equal.[11,12]. Nature recommends to ingest fatty acids in a balanced ratio (polyunsaturated: saturated $=\mathrm{w}-6: \mathrm{w}-3=1: 1$ )as part of dietary lipid pattern in monounsaturated fatty acids (P:M: $\mathrm{S}=1: 6: 1)$. These ratios represent the overall distribution of fats in a natural untamed environment. (www.columbusconcept.com). The Columbus foods include egg, milk, meat, oil , and bread, all rich in w-3 fatty acids, similar to wild foods, consumed about 160 years ago from now. Blood lipid composition does reflect one's health status: (a) circulating serum lipoproteins and their ratio provide information on their atherogenicity to blood vessels and (b) circulating plasma fatty acids, such as w-6/w-3 fatty acid ratio, give indication on proinflammatory status of blood vessels.; (a) and (b) are phenotype-related and depend on genetic, environmental and developmental factors. As such, they appear as universal markers for holistic health. Blood cholesterol is central to this approach. Its 3D-representation shows how circulating lipoproteins affect blood vessels integrity upon their circulating throughout the body. Of major importance appear the essential dietary nutrients (essential amino acids, fatty acids, antioxidant vitamins and minerals) and the functional component of the regimen (diet, sport, spiritualism, etc).The Ying Yang Tsim Tsoum (YYTT) Concept is an extension of the Columbus Concept (YYCC). While YYCC is a 1-D approach that establishes the basis for the "essential" components of the diet YYTT is a 2-D approach that focuses on the "functional" components of the lifestyle; stress and strain, sedentary behaviour, meditation. YYCC and YYTT are complementary and do not compete against each other. Chronobiology is an important aspect of YYTT because it is based on mind- brain- body interactions.

(http://www.tsimtsoum.net/introduction_07.php). It seems that the YYTT approach favors the analysis of memes and genes as well as of (hu) man's influence on nature and also possibly the influence of nurture and environment on human health and behaviour. In several studies, breathing exercises, meditation and yoga have been found to influence brain function, cardiovascular function as well as other biomarkers of health and diseases. There is evidence [11, 12] that $\omega 3$ PUFAs play an important role in neuronal structure and function. The brain is quite rich in $\omega 3$ PUFAs and several studies suggest a role for $\omega 3$ PUFAs in neurotransmitter synthesis, degradation, release, reuptake and binding [26]. Fatty acids belong to the phospholipid group and, consequently, are part of all biological membranes. The membrane's fluidity, which is of crucial importance for its functioning, depends on its lipid components. Phospholipids built up of chains of polyunsatured fatty acids increase the membrane fluidity because, by binding some chains, double bonds prevent them from compacting themselves perfectly. In addition, membrane fluidity is determined by the phospholipids/free cholesterol ratio, as cholesterol increases membrane viscosity. DHA deficit is associated with dysfunctions of neuronal membrane stability and transmission of serotonin, norepinephrine and dopamine, which might be related to the aetiology of the mood and cognitive dysfunction of depression [26]. On the other hand, EPA is essential to balance the immune function and physical health by reducing the propor-

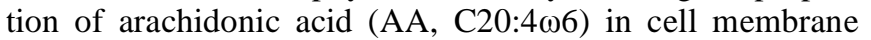
and prostaglandin E2 (PGE2) synthesis. A diet based on a high proportion of essential polyunsatured fatty acids allows a higher incorporation of cholesterol in the membranes to balance their fluidity, which, in turn, would contribute to lower blood cholesterol levels and inflammation and lower risk of CVD, osteoporosis and neuro-psychological disorders [20-26]. These interactions of dietneurotransmitters, neuronal function and other body functions and dysfunctions provide a basis for YYTT concept and the mind-body connection.

In brief, it is possible that increased consumption of proinflammatory foods may be associated with inflammation which may predispose CVD as well as osteoporotic fractures as well as depression and depentia. Increase in inflammation may explain the pathogenesis of increased rates of hip fractures after developing CVD. Increased consumption of fruits, vegetables, legumes, nuts, yogurt, canola oil or mustered oil may be protective against CVDs as well as hip fractures and body-mind dysfunctions.

\section{ACKNOWLEDGEMENTS}

Acknowledgements are due to Tsim Tsoum Institute and International College of Nutrition for support to present this study. 


\section{REFERENCES}

[1] Lau EM, Coopper C. The epidemiology of osteoporosis: the oriental perspective in a world context. Clin Othop Retal Res 1996; 323: 65-74.

[2] Cooper C, Campion G, Melton LJ. Hip fractures in the elderly: a world-wide projection. Osteoporos Int 1992; 2: 285-9.

[3] Johnell C. The socioeconomic burden of fractures: today and in the $21^{\text {st }}$ century. Am J Med 1997; 103(Suppl 2A): 20-5.

[4] Report of a Joint WHO/FAO Expert Consultation. Diet, Nutrition and the Prevention of Chronic Diseases. WHO, Geneva 2003, pp. 129-33.

[5] Sennerby Ulf, Melhus H, Gedeborg R, et al. Cardiovascular diseases and risk of hip fracture. JAMA 2009; 302: 1666-73.

[6] Kartikey K, Singh G, Kidyore, et al. Association of dietary w-6/w3 fatty acid ratio and inflammation with risk of hip fracture. Open Nutra J 2009 (In press).

[7] de Lorgeril M, Renaud S, Mamelle N, et al. Mediterranean alphalinolenic acid-rich diet in secondary prevention of coronary heart disease. Lancet 1994; 343: 1454-9.

[8] Renaud S, de Lorgeril M, Delaye J, et al. Cretan Mediterranean diet for prevention of coronary heart disease. Am J Clin Nutr 1995; 61(6 Suppl): 1360S-7S

[9] Singh RB, DeMeester F, Mechirova V, Pella D, Otsuka K. Fatty acids in the causation and therapy of metabolic syndrome. In: DeMeester F, Watson RR, Eds. Wild type foods in health promotion and disease prevention. New Jersey: Humana Press 2008; pp. 263-84.

[10] Singh RB, IL Suh, VP Singh, et al. Hypertension and stroke in Asia: prevelance, control and strategies in developing countries for prevention. J Hum Hypertens 2000; 14: 749-63.

[11] Simopoulos AP. Importance of the ratio of omega-6/omega-3 essential fatty acids: evolutionary aspects. World Rev Nutr Diet 2003; 92: 1-22.

[12] De Meester F, Wild-type land based foods in health promotion and disease prevention: the LDL-CC:HDL-CC model. In: DeMeester F, Watson RR, Eds. Wild type foods in health promotion and disease prevention. New Jersey: Humana Press 2008; pp. 3-20.

[13] Kelishadi R, Mirghaffari N, Poursafa P, Gidding SS. Lifestyle and environmental factors associated with inflammation, oxidative stress and insulin resistance in children. Atherosclerosis 2009; 203: 311-9.

[14] Sullivan DH, Nelson CL, Klimberg VS, Bopp MM. Nightly enteral nutrition support of elderly hip fracture patients: a pilot study. J Am Coll Nutr 2004; 23: 683-91.

[15] Watkins BA, Seifert MF, Allen KG. Importance of dietary fat in modulating PGE2 responses and influence of vitamin $\mathrm{E}$ on bone morphometry. World Rev Nutr Diet 1997; 82: 250-9.

[16] Watkins BA. Regulatory effects of polyunsaturates on bone remodeling and cartilage function. World Rev Nutr Diet 1998; 83: 38-51.

[17] Kruger MC, Coetzer H, de Winter R, Gericke G, van Papendrop DH. Calcium, gamma-linolenic acid and eicosapentaenoic acid supplementation in senile osteoporosis. Ageing(Milano) 1998; 10: 385-94.

[18] Terano T. Effect of omega-3 polyunsaturated fatty acid ingestion on bone metabolism and osteoporosis. World Rev Nutr Diet 2001; 88: 141-7.

[19] Vogel RA. Eating vascular biology and atherosclerosis: a lot to chew on. Eur Heart J 2006; 27: 13-4.

[20] Esposito K, Giugiliano D. Diet and inflammation: a link to metabolic and cardiovascular diseases. Eur Heart J 2006; 27: 15-20.

[21] Michelson D, Stratakis C, Hill L, et al. Bone mineral density in women with depression. N Engl J Med 1996; 335: 1176-81.

[22] Watkins BA, Li Y, Allen KG, Hoffman WE, Seifert MF. Dietary ratio of $w-6 / w-3$ polyunsaturated fatty acids alters the fatty acid composition of bone compartments and biomarkers of bone formation in rats. J Nutr 2000; 130: 2274-84.

[23] Watkins BA, Shen CL, Allen K, Seifert MF. Dietary w-3 and w-6 polyunsaturates and acetyle salicyclic acid alter ex vivo PGE2 biosynthesis, tissue IGF1 levels and bone morphometry in chicks. J Bone Miner Res 1996; 11: 1321-32.

[24] Atkinson TG, Barker HJ, Mechling Gill KA. Incorporation of long chain w-3 fatty acids in tissues and enhanced bone marrow cellularity with docosahexaenoic acid feeding in post weaning. Fischers Lipids 1997; 32: 293-302.

[25] Tashjian AH, Voelkel EF, Lazzaro M, Goad D, Bosma T, Levine L. Tumor necrosis factor-alpha(cachectin) stimulates bone reabsorption in mouse calvaria via a prostaglandin mediated mechanism. Endocrinology 1997; 120: 2029-36.

[26] Crawford MA, Bazinet RP, Sinclair AJ. Fat intake and CNS functioning: Ageing and disease. Ann Nutr Metab 2009; 55: 202-28.

(C) Kartikey et al.; Licensee Bentham Open.

This is an open access article licensed under the terms of the Creative Commons Attribution Non-Commercial License (http://creativecommons.org/licenses/ by-nc/3.0/) which permits unrestricted, non-commercial use, distribution and reproduction in any medium, provided the work is properly cited. 